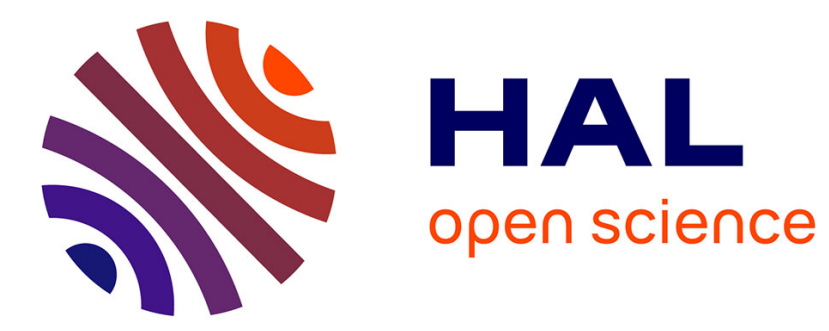

\title{
Inter-Criteria Analysis Based on Belief Functions for GPS Surveying Problems
}

\author{
Stefka Fidanova, Jean Dezert, Albena Tchamova
}

\section{To cite this version:}

Stefka Fidanova, Jean Dezert, Albena Tchamova. Inter-Criteria Analysis Based on Belief Functions for GPS Surveying Problems. IEEE INISTA 2019, Jul 2019, SOFIA, Bulgaria. hal-02355690

\section{HAL Id: hal-02355690 \\ https://hal.science/hal-02355690}

Submitted on 8 Nov 2019

HAL is a multi-disciplinary open access archive for the deposit and dissemination of scientific research documents, whether they are published or not. The documents may come from teaching and research institutions in France or abroad, or from public or private research centers.
L'archive ouverte pluridisciplinaire HAL, est destinée au dépôt et à la diffusion de documents scientifiques de niveau recherche, publiés ou non, émanant des établissements d'enseignement et de recherche français ou étrangers, des laboratoires publics ou privés. 


\section{Inter-Criteria Analysis Based on Belief Functions for GPS Surveying Problems}

\author{
Stefka Fidanova \\ Inst.of Information and Comm. Tech. \\ Bulg. Acad. of Sciences, Sofia, Bulgaria. \\ stefka@parallel.bas.bg
}

\author{
Jean Dezert \\ The French Aerospace Lab \\ Palaiseau, France. \\ jean.dezert@onera.fr
}

\author{
Albena Tchamova \\ Inst.of Information and Comm. Tech. \\ Bulg. Acad. of Sciences, Sofia, Bulgaria. \\ tchamova@bas.bg
}

\begin{abstract}
In this paper we present an application of a new Belief Function-based Inter-Criteria Analysis (BF-ICrA) approach for Global Positioning System (GPS) Surveying Problems (GSP). GPS surveying is an NP-hard problem. For designing Global Positioning System surveying network, a given set of earth points must be observed consecutively. The survey cost is the sum of the distances to go from one point to another one. This kind of problems is hard to be solved with traditional numerical methods. In this paper we use BF- ICrA to analyze an Ant Colony Optimization (ACO) algorithm developed to provide near-optimal solutions for Global Positioning System surveying problem.
\end{abstract}

Index Terms-Inter-Criteria Analysis, BF-ICrA, GPS surveying, belief functions.

\section{INTRODUCTION}

In our previous work [1] we did apply classical Atanassov's Inter-Criteria Analysis (ICrA) to examine some relations between considered GSP's and ACO algorithm performance. In this paper we consider a recent improved version of ICrA based on belief functions [2] and show how to apply it in same GSP problematic to revise and refine our previous analysis.

After a short presentation of GSP problematic and ACO in the next section, and brief basics of BF in section III, we recall the classical Atanassov's ICrA method in section IV and we present the new ICrA method based on Belief Functions, called BF-ICrA, in section V. In section VI, we show how to apply BF-ICrA for GSP problematic. Concluding remarks are given in Section VII.

\section{Presentation of ACO And GSP PRoblematic}

\section{A. GPS surveying problem description}

GPS satellites continuously transmit radio signals to the Earth while orbiting it. A receiver, with unknown position on Earth, has to detect and convert the signals received from all of the satellites into useful measurements. These measurements would allow a user to compute a three-dimensional coordinate position: location of the receiver. Any GPS observation is proven to have biases, hence, in order to survey an appropriate combination of measurement processing strategies must be used to minimize their effect on the positioning results. Differencing data collected simultaneously from two or more GPS receivers to several GPS satellites allows to eliminate

978-1-7281-1862-8/19/\$31.00 (C)2019 European Union or significantly reduce most of the biases. The GPS network can be defined as set of stations $\left(a_{1}, a_{2}, \ldots a_{n}\right)$, which are co-ordinated by placing receivers $(X 1, X 2, \ldots)$ on them to determine sessions $\left(a_{1} a_{2}, a_{1} a_{3}, a_{2} a_{3}, \ldots\right)$ among them. The problem is to search for the best order in which these sessions can be organized to give the best schedule. Thus, the schedule can be defined as a sequence of sessions to be observed consecutively. The solution is represented by linear graph with weighted edges. The nodes represent the stations and the edges represent the moving cost. The objective function of the problem is the cost of the solution which is the sum of the costs (time) to move from one point to another one, $C(V)=\sum C\left(a_{i}, a_{j}\right)$, where $a_{i} a_{j}$ is a session in solution $V$. For example if the number of points (stations) is 4 , a possible solution is $V=\left(a_{1}, a_{3}, a_{2}, a_{4}\right)$ and it can be represented by linear graph $a_{1} \rightarrow a_{3} \rightarrow a_{2} \rightarrow a_{4}$. The moving costs are as follows: $C\left(a_{1}, a_{3}\right), C\left(a_{3}, a_{2}\right), C\left(a_{2}, a_{4}\right)$. Thus the cost of the solution is $C(V)=C\left(a_{1}, a_{3}\right)+C\left(a_{3}, a_{2}\right)+C\left(a_{2}, a_{4}\right)$. In practice, determining how each GPS receiver should be moved between stations to be surveyed in an efficient manner taking into account some important factors such as time, cost etc. The problem is to search for the best order, with respect to the time, in which these sessions can be observed to give the cheapest schedule or to minimize $C(V)$. The initial data is a cost matrix, which represents the cost (time, or distance) of moving a receiver from one point to another. Solving such problems GSPs - to optimality requires a very high computational time. Therefore, meta-heuristic methods are used to provide nearoptimal solutions for large networks within acceptable amount of computational effort. In this paper, we consider the MaxMin Ant System (MMAS) meta-heuristic [3] and we present it briefly in the next subsection.

\section{B. Ant colony optimization for GPS surveying problem}

Real ants foraging for food lay down quantities of pheromone (chemical cues) marking the path that they follow. An isolated ant moves essentially at random but an ant encountering a previously laid pheromone will detect it and decide to follow it with high probability and thereby reinforce it with a further quantity of pheromone. The repetition of the above mechanism represents the auto-catalytic behavior of real ant colony where the more the ants follow a trail, the more attractive that trail becomes. 
The ACO algorithm uses a colony of artificial ants that behave as cooperative agents in a mathematics space were they are allowed to search and reinforce pathways (solutions) in order to find the optimal ones. The problem is represented by graph and the ants walk on the graph to construct solutions. The solution is represented by path in the graph. After initialization of the pheromone trails, ants construct feasible solutions, starting from random nodes, then the pheromone trails are updated. At each step ants compute a set of feasible moves and select the best one (according to some probabilistic rules) to carry out the rest of the tour. The transition probability $p_{i j}$, to chose the node $j$ when the current node is $i$, is based on the heuristic information $\eta_{i j}$ and pheromone trail level $\tau_{i j}$ of the move, where $i, j=1, \ldots, n$.

$$
p_{i j}=\frac{\tau_{i j}^{\alpha} \eta_{i j}^{\beta}}{\sum_{k \in \text { Unused }} \tau_{i k}^{\alpha} \eta_{i k}^{\beta}} .
$$

The higher value of the pheromone and the heuristic information, the more profitable is to select this move and resume the search. In the beginning, the initial pheromone level is set to a small positive constant value $\tau_{0}$ and then ants update this value after completing the construction stage. ACO algorithms adopt different criteria to update the pheromone level.

In our implementation we use MAX-MIN Ant System (MMAS) [3], [4], which is ones of the best ant approaches. In MMAS the main is using fixed upper bound $\tau_{\max }$ and lower bound $\tau_{\min }$ of the pheromone trails. Thus accumulation of big amount of pheromone by part of the possible movements and repetition of same solutions is partially prevented. The main features of MMAS are:

The aim of using only one solution is to make solution elements, which frequently occur in the best found solutions, get large reinforcement. Pheromone trail update is given by:

$$
\tau_{i j} \leftarrow \rho \tau_{i j}+\Delta \tau_{i j}
$$

where

$$
\Delta \tau_{i j}= \begin{cases}1 / C\left(V_{\text {best }}\right) & \text { if }(i, j) \in \text { best solution } \\ 0 & \text { otherwise }\end{cases}
$$

and $V_{\text {best }}$ is the iteration best solution and $i, j=1, \ldots, n$.

To avoid stagnation of the search, the range of possible pheromone value on each movement is limited to an interval $\left[\tau_{\min }, \tau_{\max }\right] . \tau_{\max }$ is an asymptotic maximum of $\tau_{i j}$ and $\tau_{\max }=1 /(1-\rho) C\left(V^{*}\right)$, while $\tau_{\min }=0.087 \tau_{\max }$. Where $V^{*}$ is the optimal solution, but it is unknown, therefore we use $V_{\text {best }}$ instead of $V^{*}$.

When all ants have completed their solutions, the pheromone level is updated by applying the global update rule. Only the pheromone corresponding to the best found solution is increased by the similar to the MMAS way. The global update rule is intended to provide a greater amount of pheromone on the paths of the best solution. It is a kind of intensification of the search around the best found solution.

We use heuristic information equals to one over the cost of the session.

\section{BASICS OF THE THEORY OF BELIEF FUNCTIONS}

Let consider a finite discrete frame of discernement (FoD) $\Theta=\left\{\theta_{1}, \theta_{2}, \ldots, \theta_{n}\right\}$, with $n>1$, and where $\theta_{i} \cap \theta_{j}=\emptyset$ for $i \neq j$. The power-set of $\Theta$ (i;e. the set of all subsets of $\Theta$ ) is denoted $2^{\Theta}$. A basic belief assignment (BBA) associated with a given source of evidence is defined [5] as the mapping $m(\cdot): 2^{\Theta} \rightarrow[0,1]$ satisfying $m(\emptyset)=0$ and $\sum_{A \in 2^{\Theta}} m(A)=$ 1. The quantity $m(A)$ is called the mass of $A$ committed by the source of evidence. Belief and plausibility functions are usually interpreted respectively as lower and upper bounds of unknown (possibly subjective) probability measure [6]. They are defined by ${ }^{1}$

$$
\operatorname{Bel}(A) \triangleq \sum_{B \subseteq A, B \in 2^{\Theta}} m(B), \quad \text { and } \quad P l(A) \triangleq 1-\operatorname{Bel}(\bar{A}) .
$$

If $m(A)>0, A$ is called a focal element of $m(\cdot)$. When all focal elements are singletons then $m(\cdot)$ is called a Bayesian $B B A$ and its corresponding $\mathrm{Bel}(\cdot)$ function is homogeneous to a probability measure. Historically the combination of BBAs is accomplished by Dempster's rule in Dempster-Shafer Theory (DST) [5]. Because of serious problems of Dempster's rule ${ }^{2}$, we recommend the Proportional Conflict Redistribution rule no. 6 (PCR6) proposed by Martin and Osswald in [10] (Vol. 3) which remains the most appealing alternative rule for BBA combination so far.

\section{Atanassov's Inter-Criteria AnAlysis (ICRA)}

Based on Intuitionistic Fuzzy Sets (IFS) [11], the InterCriteria Analysis (ICrA) has been introduced in 2014 by Atanassov et al. in [12], and then improved in [13], [15]. ICrA aims to identify the possible links between the criteria involved in a process of evaluation of multiple objects against multiple criteria. The aim of ICrA is to discover any existing correlations between the criteria themselves. Such analysis can permit (when possible) to reduce the complexity of large multiple criteria decision-making (MCDM) problems [2]. Until now the classical ${ }^{3} \mathrm{ICrA}$ has been applied in different fields: medicine [16], [17], optimization [18]-[21], workforce planning [22], competitiveness analysis [23], radar detection [24], ranking [25]-[27], etc. In this section we just recall the basic principles of classical ICrA.

Let consider a set of alternatives (or objects) $\mathbf{A} \triangleq$ $\left\{A_{1}, A_{2}, \ldots, A_{M}\right\}(M>2)$, and a set of criteria $\mathbf{C} \triangleq$ $\left\{C_{1}, C_{2}, \ldots, C_{N}\right\}(N \geq 1)$. The available information is expressed by a $M \times N$ score matrix ${ }^{4} \mathbf{S} \triangleq\left[S_{i j}=C_{j}\left(A_{i}\right)\right]$, and (eventually) the importance factor $w_{j} \in[0,1]$ of each criterion $C_{j}$ with $\sum_{j=1}^{N} w_{j}=1$. The ICrA method consists to build an $N \times N$ Inter-Criteria (IC) matrix $\mathbf{K}$ from the score matrix $\mathbf{S}$. The elements of the IC matrix $\mathbf{K}$ consist of all Intuitionistic Fuzzy (IF) pairs $\left(\mu_{j j^{\prime}}, \nu_{j j^{\prime}}\right) \in[0,1] \times[0,1]$ whose

\footnotetext{
${ }^{1}$ In the notations, the symbol $\triangleq$ means equal by definition.

${ }^{2}$ that is: 1) insensitivity to the level of conflict between sources in some cases and dictatorial behavior [7], [8], and 2) inconsistency of Shafer's belief conditioning [9] with bounds of conditional probabilities.

${ }^{3}$ We refer Atanassov's ICrA as the classical approach in the sequel.

${ }^{4}$ also called benefit or payoff matrix in Multi-Criteria Decision-Making framework.
} 
components express respectively the degree of agreement and the degree of disagreement between criteria $C_{j}$ and $C_{j^{\prime}}$ for $j, j^{\prime} \in\{1,2, \ldots, N\}$. For a given column $j$ (i.e. criterion $C_{j}$ ), it is always possible to compare with $>,<$ and $=$ operators all the scores $S_{i j}$ for $i=1,2, \ldots M$ because the scores of each column are expressed in same unit. The construction of IC matrix $\mathbf{K}$ can be used to search relations between the criteria because the method compares homogeneous data relatively to a same column. Atanassov in [14] prescribes $^{5}$ the normalization of the element $S_{i j}$ of score matrix $\mathbf{S}$ by taking

$$
S_{i j}^{\text {norm }}=\left(S_{i j}-S_{j}^{\text {min }}\right) /\left(S_{j}^{\max }-S_{j}^{\min }\right) \in[0,1]
$$

where

$$
\left\{\begin{array}{l}
S_{j}^{\min }=\min \left\{S_{1 j}, \ldots, S_{M j}\right\} \\
S_{j}^{\max }=\max \left\{S_{1 j}, \ldots, S_{M j}\right\}
\end{array}\right.
$$

The construction of the $N \times N$ IC matrix $\mathbf{K}$ is based on the pairwise comparisons between every two criteria along all evaluated alternatives. More precisely in [14] the degree of agreement between criteria $C_{j}$ and $C_{j^{\prime}} \mu_{j j^{\prime}}$, and their degree of disagreement $\nu_{j j^{\prime}}$ are calculated by

$$
\mu_{j j^{\prime}} \triangleq \frac{2 K_{j j^{\prime}}^{\mu}}{M(M-1)} \quad \text { and } \quad \nu_{j j^{\prime}} \triangleq \frac{2 K_{j j^{\prime}}^{\nu}}{M(M-1)}
$$

where $K_{j j^{\prime}}^{\mu}$ be the number of cases in which the inequalities $S_{i j}>S_{i^{\prime} j}$ and $S_{i j^{\prime}}>S_{i^{\prime} j^{\prime}}$ hold simultaneously, and $K_{j j^{\prime}}^{\nu}$ be the number of cases in which the inequalities $S_{i j}>S_{i^{\prime} j}$ and $S_{i j^{\prime}}<S_{i^{\prime} j^{\prime}}$ hold simultaneously.

By construction the IC matrix $\mathbf{K}$ is always a symmetric matrix. Atanassov provides explicit formulas in [14] for $K_{j j^{\prime}}^{\mu}$ and $K_{j j^{\prime}}^{\nu}$ which depend on a particular choice of the signum function. Because of this the results of $K_{j j^{\prime}}^{\mu}$ and $K_{j j^{\prime}}^{\nu}$ are disputable and that is why some authors [22], [28] propose other methods to calculate $K_{j j^{\prime}}^{\mu}$ and $K_{j j^{\prime}}^{\nu}$ values for making the Inter-Criteria Analysis.

Once the IC matrix $\mathbf{K}=\left[K_{j j^{\prime}}\right]$ of intuitionistic fuzzy pairs is calculated one needs to analyze it to decide which criteria $C_{j}$ and $C_{j^{\prime}}$ are in strong agreement (or positive consonance) reflecting the correlation between $C_{j}$ and $C_{j^{\prime}}$, in strong disagreement (or negative consonance) reflecting non correlation between $C_{j}$ and $C_{j^{\prime}}$, or in dissonance reflecting the uncertainty situation where nothing can be said about the non correlation or the correlation between $C_{j}$ and $C_{j^{\prime}}$.

At the beginning of ICrA development it was not very clear how these intuitionistic fuzzy (IF) pairs $\left(\mu_{j j^{\prime}}, \nu_{j j^{\prime}}\right)$ had to be used and that is why Atanassova [29], [30] proposed to handle both components of the IF pair. For this, she interpreted pairs $\left(\mu_{j j^{\prime}}, \nu_{j j^{\prime}}\right)$ as points located in the elementary $T F U$ triangle, where the point $T$ of coordinate $(1,0)$ represents the maximal positive consonance (i.e. the true consonance), the point $F$ with coordinate $(0,1)$ represents the maximal negative consonance (i.e. the falsity), and the point $U$ with coordinates $(0,0)$ represents the maximal dissonance (i.e. the uncertainty). From this interpretation it becomes quite easy to identify the

\footnotetext{
${ }^{5}$ Although this normalization is not very necessary in fact for ICrA making.
}

top of consonant IF pairs $\left(\mu_{j j^{\prime}}, \nu_{j j^{\prime}}\right)$ that fall in bottom right corner of $(T F U)$ triangle limited by vertical line from $x$-axis $x=\alpha$, and horizontal line from $y$-axis $y=\beta$, where $\alpha$ and $\beta$ are two ad-hoc threshold values in $[0,1]$. The set of consonant IF pairs are then ranked according to their (Euclidean) distance $d_{C_{j} C_{j^{\prime}}}^{T}$ with respect to $T$ point of coordinate $(1,0)$ defined by

$$
d_{C_{j} C_{j^{\prime}}}^{T}=d\left((1,0),\left(\mu_{j j^{\prime}}, \nu_{j j^{\prime}}\right)\right)=\sqrt{\left(1-\mu_{j j^{\prime}}\right)^{2}+\nu_{j j^{\prime}}^{2}}
$$

It is worth noting that $\mu_{j j^{\prime}}$ and $\nu_{j j^{\prime}}$ values are in fact linked with belief function through the following formulas

$$
\begin{aligned}
& \operatorname{Bel}_{j j^{\prime}}(\theta)=\mu_{j j^{\prime}} \\
& P l_{j j^{\prime}}(\theta)=1-\nu_{j j^{\prime}} \\
& U_{j j^{\prime}}(\theta)=P l_{j j^{\prime}}(\theta)-B e l_{j j^{\prime}}(\theta)=1-\nu_{j j^{\prime}}-\mu_{j j^{\prime}}
\end{aligned}
$$

where $\theta$ means: the criteria $C_{j}$ and $C_{j^{\prime}}$ are totally positively consonant (i.e. totally correlated), whereas $\bar{\theta}$ means: the criteria $C_{j}$ and $C_{j^{\prime}}$ are totally negatively consonant (uncorrelated). The FoD is defined as $\Theta \triangleq\{\theta, \bar{\theta}\}$. $U_{j j^{\prime}}(\theta)$ represents the dissonance (the uncertainty about the correlation) of the criteria $C_{j}$ and $C_{j^{\prime}}$. From this, one can easily define any BBA $m_{j j^{\prime}}(\theta), m_{j j^{\prime}}(\bar{\theta})$ and $m_{j j^{\prime}}(\theta \cup \bar{\theta})$ of $2^{\Theta}$ by taking

$$
\begin{aligned}
& m_{j j^{\prime}}(\theta)=\mu_{j j^{\prime}} \\
& m_{j j^{\prime}}(\bar{\theta})=\nu_{j j^{\prime}} \\
& m_{j j^{\prime}}(\theta \cup \bar{\theta})=1-\mu_{j j^{\prime}}-\nu_{j j^{\prime}}
\end{aligned}
$$

Remark 1: The construction of the Inter-Criteria Matrix $\mathbf{K}$ is not unique and depends on the choice of algorithm of construction of $\mu_{j j^{\prime}}$ and $\nu_{j j^{\prime}}$ (and the choice of the signum function) as reported in [28]. This can yield different ICrA results in general.

Remark 2: The construction of $\mu_{j j^{\prime}}$ and $\nu_{j j^{\prime}}$ appears to be only a crude approximation of true values because they are only based on counting the valid " $>$ " or " $<$ " inequalities. In fact, their calculations do not exploit how bigger and how smaller the scores values are in each comparison done. So it yields a lack of precision on estimation of $\mu_{j j^{\prime}}$ and $\nu_{j j^{\prime}}$ values.

ICrA can be very useful for verification of algorithm correctness. When the optimization problem have a lot of constraints with ICrA we can find if some of the constrain is subconstrain of some other and to exclude it. With the help of ICrA we can divide constraints to two or more groups, more sensitive and less sensitive and to solve problem first according more sensitive constraints and later to less sensitive ones. To circumvent the aforementioned drawbacks, we present succinctly in the next section a new ICrA approach based on belief functions which is presented in more details with examples in [2].

\section{A NEW ICRA METHOD BASED ON BELIEF FUNCTIONS}

The new Belief Function based ICrA method, called BFICrA for short, presented in this section improves Atanassov's ICrA. It provides a more precise construction of $\mu_{j j^{\prime}}$ and $\nu_{j j^{\prime}}$ values because it exploits all available information included in the score matrix. 
BF-ICrA starts with the construction of an $M \times N$ BBA matrix $\mathbf{M}=\left[m_{i j}(\cdot)\right]$ from the score matrix $\mathbf{S}=\left[S_{i j}\right]$. The BBA matrix $\mathbf{M}$ is obtained as follows - see [31] for details and justification.

$$
\begin{aligned}
m_{i j}\left(A_{i}\right) & =\operatorname{Bel}_{i j}\left(A_{i}\right) \\
m_{i j}\left(\bar{A}_{i}\right) & =\operatorname{Bel}_{i j}\left(\bar{A}_{i}\right)=1-P l_{i j}\left(A_{i}\right) \\
m_{i j}\left(A_{i} \cup \bar{A}_{i}\right) & =P l_{i j}\left(A_{i}\right)-\operatorname{Bel}_{i j}\left(A_{i}\right)
\end{aligned}
$$

where $^{6}$

$$
\begin{aligned}
& \operatorname{Bel}_{i j}\left(A_{i}\right) \triangleq \operatorname{Sup}_{j}\left(A_{i}\right) / A_{\max }^{j} \\
& \operatorname{Bel}_{i j}\left(\bar{A}_{i}\right) \triangleq \operatorname{Inf}_{j}\left(A_{i}\right) / A_{\text {min }}^{j}
\end{aligned}
$$

with

$$
\begin{aligned}
& \operatorname{Sup}_{j}\left(A_{i}\right) \triangleq \sum_{k \in\{1, \ldots M\} \mid S_{k j} \leq S_{i j}}\left|S_{i j}-S_{k j}\right| \\
& \operatorname{Inf}_{j}\left(A_{i}\right) \triangleq-\sum_{k \in\{1, \ldots M\} \mid S_{k j} \geq S_{i j}}\left|S_{i j}-S_{k j}\right|
\end{aligned}
$$

and

$$
\begin{aligned}
& A_{\max }^{j} \triangleq \max _{i} \operatorname{Sup}_{j}\left(A_{i}\right) \\
& A_{\min }^{j} \triangleq \min _{i} \operatorname{Inf}_{j}\left(A_{i}\right)
\end{aligned}
$$

For another criterion $C_{j^{\prime}}$ and the $j^{\prime}$-th column of the score matrix we will obtain another set of BBA values $m_{i j^{\prime}}(\cdot)$. Applying this method for each column of the score matrix we are able to compute the BBA matrix $\mathbf{M}=\left[m_{i j}(\cdot)\right]$ whose each component is in fact a triplet $\left(m_{i j}\left(A_{i}\right), m_{i j}\left(\bar{A}_{i}\right), m_{i j}\left(A_{i} \cup\right.\right.$ $\left.\bar{A}_{i}\right)$ ) of BBA values in $[0,1]$ such that $m_{i j}\left(A_{i}\right)+m_{i j}\left(\bar{A}_{i}\right)+$ $\left.m_{i j}\left(A_{i} \cup \bar{A}_{i}\right)\right)=1$ for all $i=1, \ldots, M$ and $j=1, \ldots, N$.

The next step of BF-ICrA approach is the construction of the $N \times N$ Inter-Criteria Matrix $\mathbf{K}=\left[K_{j j^{\prime}}\right]$ from $M \times N$ BBA matrix $\mathbf{M}=\left[m_{i j}(\cdot)\right]$ where elements $K_{j j^{\prime}}$ corresponds to the $\operatorname{BBA}\left(m_{j j^{\prime}}(\theta), m_{j j^{\prime}}(\bar{\theta}), m_{j j^{\prime}}(\theta \cup \bar{\theta})\right)$ about positive consonance $\theta$, negative consonance $\theta$ and uncertainty between criteria $C_{j}$ and $C_{j^{\prime}}$ respectively. The construction of the triplet $K_{j j^{\prime}}=\left(m_{j j^{\prime}}(\theta), m_{j j^{\prime}}(\bar{\theta}), m_{j j^{\prime}}(\theta \cup \bar{\theta})\right)$ is based on two steps:

- Step 1 (BBA construction): Getting $m_{j j^{\prime}}^{i}$ (.).

For each alternative $A_{i}$ for $i=1, \ldots, M$, we first compute the BBA $\left(m_{j j^{\prime}}^{i}(\theta), m_{j j^{\prime}}^{i}(\bar{\theta}), m_{j j^{\prime}}^{i}(\theta \cup\right.$ $\bar{\theta})$ ) for any two criteria $j, j^{\prime} \in\{1,2, \ldots, N\}$. For this, we consider two sources of evidences (SoE) indexed by $j$ and $j^{\prime}$ providing the BBA $m_{i j}$ and $m_{i j^{\prime}}$ defined on the simple FoD $\left\{A_{i}, \bar{A}_{i}\right\}$ and denoted $m_{i j}=\left[m_{i j}\left(A_{i}\right), m_{i j}\left(\bar{A}_{i}\right), m_{i j}\left(A_{i} \cup \bar{A}_{i}\right)\right]$ and $m_{i j^{\prime}}=$ $\left[m_{i j^{\prime}}\left(A_{i}\right), m_{i j^{\prime}}\left(\bar{A}_{i}\right), m_{i j^{\prime}}\left(A_{i} \cup \bar{A}_{i}\right)\right]$. We also denote $\Theta=$ $\{\theta, \bar{\theta}\}$ the FoD about the relative state of the two SoE, where $\theta$ means that the two SoE agree, $\bar{\theta}$ means that they disagree and $\theta \cup \bar{\theta}$ means that we don't know. Hence, two SoE are in total agreement if both commit their maximum belief mass to the same element $A_{i}$ or to

\footnotetext{
${ }^{6}$ assuming that $A_{\max }^{j} \neq 0$ and $A_{\min }^{j} \neq 0$. If $A_{\max }^{j}=0$ then $\operatorname{Bel}_{i j}\left(A_{i}\right)=0$, and if $A_{\text {min }}^{j}=0$ then $P l_{i j}\left(A_{i}\right)=1$.
}

the same element $\bar{A}_{i}$. Similarly, two SoE are in total disagreement if each one commits its maximum mass of belief to one element and the other to its opposite, that is if one has $m_{i j}\left(A_{i}\right)=1$ and $m_{i j^{\prime}}\left(\bar{A}_{i}\right)=1$, or if $m_{i j}\left(\bar{A}_{i}\right)=1$ and $m_{i j^{\prime}}\left(A_{i}\right)=1$. Based on this very simple and natural principle, one can now compute the belief masses as follows:

$$
\begin{aligned}
& m_{j j^{\prime}}^{i}(\theta)=m_{i j}\left(A_{i}\right) m_{i j^{\prime}}\left(A_{i}\right)+m_{i j}(\bar{A}) m_{i j^{\prime}}(\bar{A}) \\
& m_{j j^{\prime}}^{i}(\bar{\theta})=m_{i j}\left(A_{i}\right) m_{i j^{\prime}}\left(\bar{A}_{i}\right)+m_{i j}\left(\bar{A}_{i}\right) m_{i j^{\prime}}\left(A_{i}\right) \\
& m_{j j^{\prime}}^{i}(\theta \cup \bar{\theta})=1-m_{j j^{\prime}}^{i}(\theta)-m_{j j^{\prime}}^{i}(\bar{\theta})
\end{aligned}
$$

$m_{j j^{\prime}}^{i}(\theta)$ represents the degree of agreement between the BBA $m_{i j}(\cdot)$ and $m_{i j^{\prime}}(\cdot)$ for the alternative $A_{i}, m_{j j^{\prime}}^{i}(\bar{\theta})$ represents the degree of disagreement of the two BBAs and $m_{j j^{\prime}}^{i}(\theta \cup \bar{\theta})$ the level of uncertainty (i.e. how much we don't know if they agree or disagree). By construction $m_{j j^{\prime}}^{i}(\cdot)=m_{j^{\prime} j}^{i}(\cdot), m_{j j^{\prime}}^{i}(\theta), m_{j j^{\prime}}^{i}(\bar{\theta}), m_{j j^{\prime}}^{i}(\theta \cup \bar{\theta}) \in[0,1]$ and $m_{j j^{\prime}}^{i}(\theta)+m_{j j^{\prime}}^{i}(\theta)+m_{j j^{\prime}}^{i}(\theta \cup \bar{\theta})=1$. This BBA modeling permits to build a set of $M$ symmetrical Inter-Criteria Belief Matrices (ICBM) $\mathbf{K}^{i}=\left[K_{j j^{\prime}}^{i}\right]$ of dimension $N \times N$ relative to each alternative $A_{i}$ whose components $K_{j j^{\prime}}^{i}$ correspond to the triplet of BBA values $m_{j j^{\prime}}^{i}=\left(m_{j j^{\prime}}^{i}(\theta), m_{j j^{\prime}}^{i}(\bar{\theta}), m_{j j^{\prime}}^{i}(\theta \cup \bar{\theta})\right)$ modeling the belief of agreement and of disagreement between $C_{j}$ and $C_{j^{\prime}}$ based on $A_{i}$.

- Step 2 (fusion): Getting $\mathbf{m}_{\mathbf{j} \mathbf{j}^{\prime}}($.$) .$

In this step, one needs to combine the BBAs $\mathbf{m}_{\mathbf{j} j^{\prime}}^{\mathbf{i}}($.$) for$ $i=1, \ldots, M$ altogether to get the component $K_{j j^{\prime}}=$ $\left(m_{j j^{\prime}}(\theta), m_{j j^{\prime}}(\bar{\theta}), m_{j j^{\prime}}(\theta \cup \bar{\theta})\right)$ of the Inter-Criteria Belief matrix (ICBM) $\mathbf{K}=\left[K_{j j^{\prime}}\right]$. For this, we recommend to use the PCR6 fusion rule [10] (Vol. 3) because of known deficiencies of Dempster's rule. Because of computational complexity of PCR6 fusion rule when $M$ becomes large, one may prefer to approximate the fusion result by using the simple averaging rule. Simple Matlab ${ }^{\mathrm{TM}}$ code for PCR6 rule can be found in [32] for convenience.

The computational complexity of BF-ICrA is of course higher than the complexity of ICrA because it makes a more precise evaluation of local and global inter-criteria belief matrices with respect to IF inter-criteria matrices of ICrA. The overall reduction of the computational burden of the original MCDM problem thanks to BF-ICrA depends highly on the problem under concern, the complexity and cost to evaluate each criteria involved in it, as well as the number of redundant criteria identified by BF-ICrA method.

Once the global Inter-Criteria Belief Matrix (ICBM) $\mathbf{K}=$ $\left[K_{j j^{\prime}}=\left(m_{j j^{\prime}}(\theta), m_{j j^{\prime}}(\bar{\theta}), m_{j j^{\prime}}(\theta \cup \bar{\theta})\right)\right]$ is calculated, we can identify the criteria that are in strong agreement, in strong disagreement, and those on which we are uncertain. For identifying the criteria that are in strong agreement, we evaluate the distance of each component of $K_{j j^{\prime}}$ with the BBA representing the best agreement state and characterized 
by the specific $\mathrm{BBA}^{7} m_{T}(\theta)=1$. From a similar approach we can also identify, if we want, the criteria that are in very strong disagreement using the distance of $m_{j j^{\prime}}(\cdot)$ with respect to the BBA representing the best disagreement state characterized by the specific BBA $m_{F}(\bar{\theta})=1$. We use the $d_{B I}(.,$.$) distance presented in [33] for measuring the distance$ $d\left(m_{1}, m_{2}\right)$ between the two $\mathrm{BBAs}^{8} m_{1}(\cdot)$ and $m_{2}(\cdot)$ over the same FoD. It is defined by

$$
d_{B I}\left(m_{1}, m_{2}\right) \triangleq \sqrt{N_{c} \cdot \sum_{X \in 2^{\Theta}} d_{W}^{2}\left(B I_{1}(X), B I_{2}(X)\right)}
$$

where the Belief-Intervals are defined by $B I_{1}(X) \triangleq$ $\left[B e l_{1}(X), P l_{1}(X)\right]$ and $B I_{2}(X) \triangleq\left[B e l_{2}(X), P l_{2}(X)\right]$ and computed from $m_{1}($.$) and m_{2}($.$) thanks to formula (3).$ $d_{W}\left(B I_{1}(X), B I_{2}(X)\right)$ is Wassertein's distance between intervals calculated by

$$
\begin{aligned}
& d_{W}\left(\left[a_{1}, b_{1}\right],\left[a_{2}, b_{2}\right]\right)= \\
& \sqrt{\left[\frac{a_{1}+b_{1}}{2}-\frac{a_{2}+b_{2}}{2}\right]^{2}+\frac{1}{3}\left[\frac{b_{1}-a_{1}}{2}-\frac{b_{2}-a_{2}}{2}\right]^{2}}
\end{aligned}
$$

and $N_{c}=1 / 2^{|\Theta|-1}$ is a normalization factor to get $d_{B I}\left(m_{1}, m_{2}\right) \in[0,1]$.

\section{APPliCATION OF BF-ICRA TO GSP}

In this section, we analyze the experimental results obtained using MMAS algorithm described in the previous section. For this, we use real data from Malta and Seychelles GPS networks composed of 38 sessions and 71 sessions respectively denoted GSP1 and GSP2. We use also 6 larger test problems range from 100 to 443 sessions denoted GSP3,..., GSP8. The results are obtained by performing 30 independent runs, for every experiment. The details of our MMAS implementation are given in [1]. So in our GSP example we consider 8 GSP criteria $C_{i}=G S P i, i=1, \ldots, 8$ and six average costs as results $A_{1}, \ldots, A_{6}$, where $A_{1}$ is the cost average for the first 5 runs, $A_{2}$ the cost average for the first 10 runs, $A_{3}$ for the first 15 runs), ... and finally $C_{6}$ for all the 30 runs. Table I

\begin{tabular}{|c|c|c|c|c|c|c|}
\hline & $A_{1}$ & $A_{2}$ & $A_{3}$ & $A_{4}$ & $A_{5}$ & $A_{6}$ \\
\hline$C_{1}=G S P 1$ & 899.00 & 898.00 & 898.33 & 898.50 & 899.40 & 899.50 \\
\hline$C_{2}=G S P 2$ & 916.40 & 915.60 & 922.47 & 924.80 & 924.72 & 922.07 \\
\hline$C_{3}=G S P 3$ & 41336.40 & 41052.40 & 40991.93 & 40935.90 & 40832.20 & 40910.60 \\
\hline$C_{4}=G S P 4$ & 3244.80 & 3303.30 & 3327.00 & 3344.55 & 3345.60 & 3341.93 \\
\hline$C_{5}^{4}=G S P 5$ & 1656.20 & 1660.80 & 1663.93 & 1664.95 & 1666.96 & 1665.90 \\
\hline$C_{6}=G S P 6$ & 1673.60 & 1683.50 & 1690.73 & 1688.75 & 1690.24 & 1692.67 \\
\hline$C_{7}=G S P 7$ & 3420.00 & 3430.70 & 3433.13 & 3426.85 & 3429.44 & 3428.57 \\
\hline$C_{8}=G S P 8$ & 3758.20 & 3755.70 & 3758.73 & 3760.50 & 3760.80 & 3765.80 \\
\hline
\end{tabular}
shows the values of averaged costs obtained for this problem. It corresponds to the transpose of the score matrix $\mathbf{S}$.

Hence in this problem $M=6$ and $N=8$, and $\mathbf{S}=\left[S_{i j}\right]$ is a $6 \times 8$ score matrix. Based on classical ICrA approach, one

\footnotetext{
${ }^{7}$ We use the index $T$ in the notation $m_{T}(\cdot)$ to refer that the agreement is true, and $F$ in $m_{F}(\cdot)$ to specify that the agreement is false.

${ }^{8}$ Here we will take $m_{1}(\cdot)=m_{j j^{\prime}}($.$) and m_{2}(\cdot)=m_{T}(\cdot)$, or $m_{2}(\cdot)=$ $m_{F}(\cdot)$
}

gets the following IC matrices ${ }^{9}$

$$
\begin{aligned}
& \begin{array}{llllllll}
C_{1} & C_{2} & C_{3} & C_{4} & C_{5} & C_{6} & C_{7} & C_{8}
\end{array} \\
& C_{1}\left[\begin{array}{cccccccc}
1 & 0.60 & 0.27 & 0.67 & 0.73 & 0.67 & 0.33 & 0.87 \\
0.60 & 1 & 0.27 & 0.80 & 0.73 & 0.53 & 0.47 & 0.73
\end{array}\right] \\
& \begin{array}{cccccccccc}
C_{3} & 0.27 & 0.27 & 1 & 0.07 & 0 & 0.20 & 0.40 & 0.73 \\
\hline
\end{array} \\
& \mathrm{K}^{\mu}=C_{4} \quad \begin{array}{c|ccccccccc}
C_{4} & 0.67 & 0.80 & 0.07 & 1 & 0.93 & 0.73 & 0.53 & 0.80 \\
C_{5} & 0.73 & 0.73 & 0 & 0.93 & 1 & 0.80 & 0.60 & 0.87
\end{array} \\
& \begin{array}{l|lllllllll}
C_{6} & 0.67 & 0.53 & 0.20 & 0.73 & 0.80 & 1 & 0.67 & 0.80
\end{array} \\
& \begin{array}{l|lllllllll}
C_{7} & 0.33 & 0.47 & 0.40 & 0.53 & 0.60 & 0.67 & 1 & 0.47
\end{array} \\
& C_{8} \quad\left[\begin{array}{lllllllll}
0.87 & 0.73 & 0.13 & 0.80 & 0.87 & 0.80 & 0.47 & 1
\end{array}\right] \\
& \begin{array}{llllllll}
C_{1} & C_{2} & C_{3} & C_{4} & C_{5} & C_{6} & C_{7} & C_{8}
\end{array} \\
& C_{1}\left[\begin{array}{cccccccc}
0 & 0.40 & 0.73 & 0.33 & 0.27 & 0.33 & 0.67 & 0.13 \\
0.40 & 0 & 0.73 & 0.20 & 0.27 & 0.47 & 0.53 & 0.27
\end{array}\right] \\
& \begin{array}{l|cccccccc}
C_{2} & 0.40 & 0 & 0.73 & 0.20 & 0.27 & 0.47 & 0.53 & 0.27 \\
C_{3} & 0.73 & 0.73 & 0 & 0.93 & 1 & 0.80 & 0.60 & 0.87
\end{array} \\
& \mathrm{~K}^{\nu}=C_{4} \quad \begin{array}{lllllllll}
C_{5} & 0.33 & 0.20 & 0.93 & 0 & 0.07 & 0.27 & 0.47 & 0.20 \\
C_{6} & 0.37 & 0.27 & 1 & 0.07 & 0 & 0.20 & 0.40 & 0.13
\end{array} \\
& \begin{array}{c|cccccccc}
C_{5} & 0.27 & 0.27 & 1 & 0.07 & 0 & 0.20 & 0.40 & 0.13 \\
C_{6} & 0.33 & 0.47 & 0.80 & 0.27 & 0.20 & 0 & 0.33 & 0.20
\end{array} \\
& \begin{array}{l|lllllllll}
C_{7} & 0.67 & 0.53 & 0.60 & 0.47 & 0.40 & 0.33 & 0 & 0.53
\end{array} \\
& C_{8} \quad\left[\begin{array}{llllllll}
0.13 & 0.27 & 0.87 & 0.20 & 0.13 & 0.20 & 0.53 & 0
\end{array}\right]
\end{aligned}
$$

The element $K_{j j^{\prime}}^{\mu}$ of matrix $\mathbf{K}^{\mu}$ expresses the degree of agreement between criteria $C_{j}=G S P_{j}$ and $C_{j^{\prime}}=G S P_{j^{\prime}}$, whereas the element $K_{j j^{\prime}}^{\nu}$ of matrix $\mathbf{K}^{\nu}$ expresses the degree of disagreement between $C_{j}=G S P_{j}$ and $C_{j^{\prime}}=G S P_{j^{\prime}}$. Based on these results, one sees that ACO algorithm performs similarly for $G S P_{2}, G S P_{4} G S P_{5}$ and $G S P_{8}$ because they are all in high agreement. Indeed $\mu_{j j^{\prime}}$ values for $j, j^{\prime} \in\{2,4,5,8\}$ are quite high (greater than $70 \%$ ). They are GPS networks with different numbers of sessions, but may have a similar structure, therefore, the value of agreement is high. For other networks, we can conclude that they have very different structure. What is worth noting is that there appears also a strong agreement of GSP1 with GSP8 because $\mu_{18}=0.87$. But because GSP8 is also in strong agreement with GSP2, GSP4, GSP5 and with GSP1 it is logically expected that GSP1 should be also in agreement with GSP2, GSP4, GSP5, which is unfortunately not the case based on this classical ICrA. This example points out some inconsistency of ICrA result because of the too crude method of estimation of the degree of agreement and disagreement between criteria based on IFS.

Now if we consider the same example with the same score matrix $\mathbf{S}$ (built from Table I), we obtain the following IC Belief matrices $^{10}$

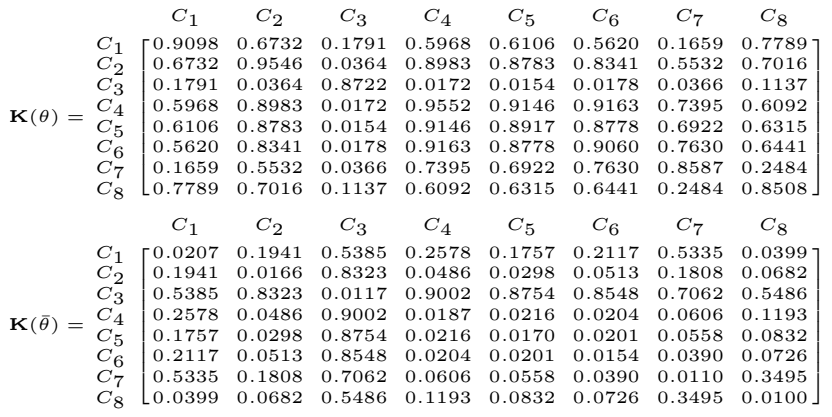

From ICBM $\mathbf{K}(\theta)$ and $\mathbf{K}(\bar{\theta})$ we compute the matrix $\mathbf{D}(\theta)$ of distance of $m_{j j^{\prime}}($.$) to the full agreement state with BBA$

${ }^{9}$ For presentation convenience and due to typesetting column width, we decompose et present the IC matrix $\mathbf{K}=\left[K_{j j^{\prime}}=\left(K_{j j^{\prime}}^{\mu}, K_{j j^{\prime}}^{\nu}\right)\right]$ into two distinct matrices $\mathbf{K}^{\mu}=\left[K_{j j^{\prime}}^{\mu}\right]$ and $\mathbf{K}^{\nu}=\left[K_{j j^{\prime}}^{\nu}\right]$.

${ }^{10}$ For presentation convenience, the ICBM $\mathbf{K}=\left[K_{j j^{\prime}}=\right.$ $\left.\left(m_{j j^{\prime}}(\theta), m_{j j^{\prime}}(\bar{\theta}), m_{j j^{\prime}}(\theta \cup \bar{\theta})\right)\right]$ is decomposed into three matrices $\mathbf{K}(\theta)=$ $\left[K_{j j^{\prime}}^{\theta}=m_{j j^{\prime}}(\theta)\right], \mathbf{K}(\bar{\theta})=\left[K_{j j^{\prime}}^{\bar{\theta}}=m_{j j^{\prime}}(\bar{\theta})\right]$ and $\mathbf{K}(\theta \cup \bar{\theta})=\left[K_{j j^{\prime}}^{\theta \cup \bar{\theta}}=\right.$ $\left.1-m_{j j^{\prime}}(\theta)-m_{j j^{\prime}}(\bar{\theta})\right]$. 
$m_{F}(\theta)=1$ based on $d_{B I}($.$) distance. We get the following$ distances to full agreement

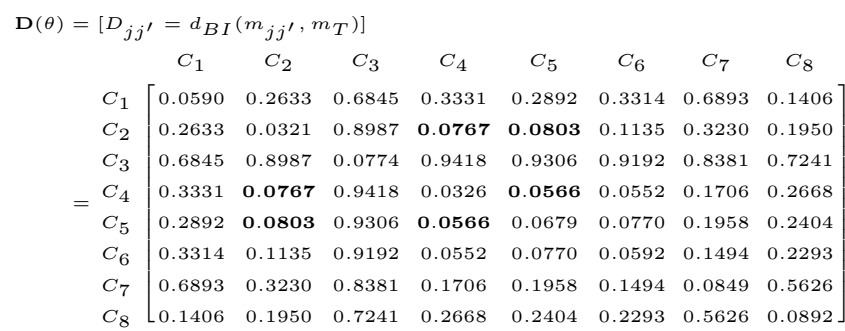

The element $D_{j j^{\prime}}$ represents the agreement distance between $C_{j}$ and $C_{j^{\prime}}$, the lower the better. From the values of elements of $\mathbf{D}(\theta)$ matrix one sees clearly that ACO performs similarly for GSP2, GSP4 and GSP5 because distances $D_{24}$, $D_{25}$, and $D_{45}$ are very small. Also we see that GSP6 is also in good agreement with GSP4 and GSP5 but is relatively less in agreement with GSP2 because $D_{26}=0.1135$. As we see there is no inconsistency in this new BF-ICrA method with respect to what provides classical ICrA because with BF-ICrA we have a much better and precise estimation of degrees of agreement and disagreement between criteria for making the analysis thanks to a proper belief functions modeling.

\section{CONCLUSION}

The GPS surveying problem and a new InterCriteria Analysis based on belief functions were addressed in this paper to overcome the potential inconsistencies of the results generated by the classical ICrA method. This technique proposes a more precise and refined method for estimating the degree of agreement and disagreement between criteria which use the whole information available in the data. Instances containing from 38 to 443 sessions have been solved using MMAS algorithm and we did compare the performance of ACO algorithms applied to eight GPS networks. Our results shows that ACO can provide fast near-optimal solution for observing GPS networks, and could help to improve the services based on GPS networks. From this new Inter-Criteria Analysis we are able to identify some relations and dependences between the considered eight GSPs and MMAS algorithm performance.

\section{ACKNOWLEDGEMENTS}

This paper is partially supported by the National Scientific Fund of Bulgaria under grant DFNI DN12/5 and by Grant No BG05M2OP001-1.001-0003, financed by the Science and Education for Smart Growth Operational Program and co-financed by the European Union through the European structural and Investment funds.

\section{REFERENCES}

[1] S. Fidanova, V. Atanassova, O. Roeva, Ant Colony Optimization Application to GPS Surveying Problems: InterCriteria Analysis. In: Atanassov $\mathrm{K}$. et al. (eds) Uncertainty and Imprecision in Decision Making and Decision Support: Cross-Fertilization, New Models and Applications. IWIFSGN 2016. Advances in Intelligent Systems and Computing, Vol 559. Springer, Cham, 2018.

[2] J. Dezert, A. Tchamova, D. Han, J.-M. Tacnet, Simplification of MultiCriteria Decision-Making Using Inter-Criteria Analysis and Belief Functions, Proc. of Fusion 2019 Int. Conf, Ottawa, Canada, July 2-5, 2019.
[3] T. Stutzle, H.H. Hoos, MAX-MIN Ant System, In: Dorigo, M., Stutzle, T., Di Caro, G. (eds.) Future Generation Computer Systems, Vol. 16, pp. 889-914, 2000.

[4] M. Dorigo, L.M. Gambardella, Ant colony system: a cooperative learning approach to the traveling salesman problem, IEEE Trans. Evol. Comput. 1, pp. 5-66, 1997.

[5] G. Shafer, A Mathematical Theory of Evidence, Princeton Press, 1976.

[6] A. Dempster, Upper and lower probabilities induced by a multivalued mapping, Ann. of Math. Stat., Vol. 38, pp. 325-339, 1967.

[7] J. Dezert, P. Wang, A. Tchamova, On The Validity of Dempster-Shafer Theory, in Proc. of Fusion 2012, Singapore, July 2012.

[8] A. Tchamova, J. Dezert, On the Behavior of Dempster's Rule of Combination and the Foundations of Dempster-Shafer Theory, IEEE IS2012, Sofia, Bulgaria, Sept. 6-8, 2012.

[9] J. Dezert, A. Tchamova, D. Han,Total Belief Theorem and Conditional Belief Functions, International Journal of Intelligent Systems, pp. 1-27, 2018.

[10] F. Smarandache, J. Dezert (Editors), Advances and applications of DSmT for information fusion, American Research Press, Vol. 1-4, 2004-2015. http://www.onera.fr/staff/jean-dezert?page $=2$

[11] K. Atanassov, On Intuitionistic Fuzzy Sets Theory, Springer, 2012.

[12] K. Atanassov, D. Mavrov, V. Atanassova, Intercriteria decision making: a new approach for multicriteria decision making, based on index matrices and intuitionistic fuzzy sets. Issues IFSs GNs 11, pp. 1-8, 2014.

[13] K. Atanassov, V. Atanassova, G. Gluhchev, InterCriteria Analysis: Ideas and problems, Notes on IFS, Vol. 21, No. 1, pp. 81-88, 2015.

[14] K. Atanassov et al., Intercriteria analysis over normalized data. Intelligent Systems (IS), 2016 IEEE 8th Int. Conference on, IEEE, 2016.

[15] K. Atanassov et al., An approach to a constructive simplification of multiagent multicriteria decision making problems via intercriteria analysis, C.R. de l'Acad. Bulgare des Sci., Vol. 70, No. 8, 2017.

[16] S. Krumova, et al., Intercriteria analysis of calorimetric data of blood serum proteome, in Bioch. et Biophys. Acta, Gen. Subjects, 1861, 2017.

[17] B. Zaharieva et al. InterCriteria decision making approach for Behterev's disease analysis, in Int. J. of Bioautom, Vol. 22(2), 2018.

[18] T. Pencheva et al., InterCriteria Analysis of Genetic Algorithm Parameters in Parameter Identification, Notes on IFS, Vol. 21(2), 2015.

[19] S. Sotirov, et al., Application of the Intuitionistic Fuzzy InterCriteria Analysis Method to a Neural Network Preprocessing Procedure, Proc of 9th EUSFLAT, pp. 1559-1564, Gijon, Spain, June 30-July 7th, 2015.

[20] M. Angelova, O. Roeva, T. Pencheva, InterCriteria analysis of crossover and mutation rates relations in simple genetic algorithm, Proc. of Conf on Computer Sci. and Inf. Syst, Vol. 5, pp. 419-424, 2015.

[21] O. Roeva, S. Fidanova, M. Paprzycki, InterCriteria analysis of ACO and GA hybrid algorithms, Stud. Comput. Intell. 610, pp. 107-126, 2016.

[22] O. Roeva et al., Intercriteria Analysis of ACO Performance for Workforce Planning Problem, Studies in Comp. Intell. 795, Springer 2019.

[23] V. Atanassova et al., Discussion on the threshold values in the InterCriteria decision making approach. Notes on Intuitionistic Fuzzy Sets 20(2), pp. 94-99, 2014.

[24] L. Doukovska, V. Atanassova, InterCriteria Analysis approach in radar detection threshold analysis, Notes on IFS, Vol. 21(4), 2015.

[25] V. Bureva et al., Application of the InterCriteria decision making method to Bulgarian universities ranking, Int. Workshop on IFSs, Bulgaria, 2015.

[26] V. Bureva, E. Sotirova, H. Panayotov, The InterCriteria decision making method to Bulgarian university ranking system, Annual of Informatics Section, Vol. 8, pp. 54-70, 2015-2016.

[27] M. Krawczak et al., Application of the InterCriteria Decision Making Method to Universities Ranking, Adv. in Intell. Syst. and Comp., Vol 401, pp. 365-372, Springer, 2016.

[28] N. Ikonomov, P. Vassilev, O. Roeva, ICrAData software for InterCriteria analysis, Int. J. BioAutomation, Vol. 22, No. 2, 2018.

[29] V. Atanassova, Interpretation in the Intuitionistic Fuzzy Triangle of the Results Obtained by the InterCriteria Analysis, Proc. of 16th World Congr. of IFSA, Atlantis Press, 2015.

[30] V. Atanassova et al., Traversing and ranking of elements of an intuitionistic fuzzy set in the intuitionistic fuzzy interpretation triangle, Adv. in Intell. Syst. and Comp., Vol. 401, pp. 161-174, 2016.

[31] J. Dezert, D.Han, H. Yin, A New Belief Function Based Approach for Multi-Criteria Decision-Making Support, Proc. of Fusion 2016 Conf.

[32] F. Smarandache, J. Dezert, J.-M. Tacnet, Fusion of sources of evidence with different importances and reliabilities, Proc.of Fusion Conf 2010.

[33] D. Han, J. Dezert, Y. Yang, New Distance Measures of Evidence based on Belief Intervals, Proc. of Belief 2014, Oxford, UK, Sept. 2014. 